\title{
Neurosyphilis Masquerading as Stroke in an 84-year-old
}

\author{
Cristina Bologa ${ }^{1}$, Catalina Lionte ${ }^{1 *}$, Diana Halit², Catalina Luca ${ }^{3}$ \\ ${ }^{1}$ Internal Medicine Department, $2^{\text {nd }}$ Internal Medicine Clinic,"Sf. Spiridon" Clinical County Emergency Hospital, “Gr. T. \\ Popa" University of Medicine, lasi, Romania \\ ${ }^{2}$ Neurology Department, "Prof. Dr. N. Oblu" Clinical Emergency Hospital, lasi, Romania \\ ${ }^{3}$ Infectious Disease Department, "Sf. Parascheva" Clinical Hospital, "Gr. T. Popa" University of Medicine, lasi, Romania
}

\begin{abstract}
The case of an 84 years old woman with uncharacteristic neurologic and cognitive symptoms, suspected of ischemic stroke is presented. Following an extensive assessment in the departments of neurology and internal medicine, the unusual aetiology of stroke was identified as meningovascular neurosyphilis. The patient fully recovered after antibiotic therapy. To our knowledge, this the eldest patient with tertiary neurosyphilis reported in the literature.
\end{abstract}

Keywords: neurosyphilis, ischemic stroke, elderly, treatment

Received: 11 January 2017 / Accepted: 13 February 2017

\section{INTRODUCTION}

Aetiology of stroke in elderly patients can be difficult to diagnose. One of the unusual etiologies of stroke is meningovascular neurosyphilis, which is frequently overlooked in the diagnosis process. We report a case of an elderly with uncharacteristic neurologic and cognitive symptoms, suspected of ischemic stroke, which required an extensive assessment in the departments of neurology and internal medicine. The patient fully recovered after antibiotic therapy.

\section{CASE PRESENTATION}

An 84 years old woman presented to the emergency department with dysarthria, weakness in her right arm, headache, dizziness, with the inability to maintain orthostatic posture. The symptoms appeared six months previously but had increased in severity in the last month. The patient had been diagnosed with hypertension several years earlier. She was undertaking chronic antihypertensive therapy with enalapril $20 \mathrm{mg} /$ day and nifedipine $20 \mathrm{mg} /$ day. She was first admitted to a county hospital, and at family's request, she was transferred to "Sf. Spiridon" Clinical County Emergency Hospital.

On admission, she had gait difficulties. She could not maintain an orthostatic position for more than seven minutes. Neurologic examination revealed: central vestibular syndrome (vertigo, nystagmus, with static and dynamic balance impairment), right incomplete hemiparesis, dysphasia, diffuse headache without meningeal irritation signs, and neuropsychiatric syndrome (insomnia, poor concentration, a deficit in attention, memory loss).

She had an area of nasal and malar hyperemia very similar to a "butterfly rash". On the nose and right retro-auricular region, she had a postoperative scar. The rest of the physical exam was normal.

The patient reported a 4-5 months' history of intermittent fever, worsening headache, dizziness, loss of appetite, and insomnia. She said she had difficulty in concentration, short-term memory loss and sometimes slurred speech. Two months previously she had had a surgical intervention for skin tumours in the right nasal region and right retro-auricular area. She could not explain the type of tumours that had been excised. She did not report any other diseases in her past medical history.

The initial CT scan of the brain excluded stroke, or cerebral vascular malformation and showed only microangiopathy-like lesions that were interpreted as secondary to poorly controlled hypertension.

Blood tests were normal as were renal function and hepatic function tests, complete blood count, fasting 
blood glucose, electrolytes and thyroid function tests. The ESR was $69 \mathrm{~mm} / 1$ hour.

To exclude vasculitis, immunological tests are necessary for a patient with neurological symptoms and hypertension. All markers of vasculitis were negative.

The level of VDRL was 1:128 and Treponema pallidum particle agglutination assay (TPPA) was more than 1:800. Cerebrospinal fluid (CSF) examination showed a protein level of $99 \mathrm{mg} / \mathrm{dl}, 29$ leukocytes $/ \mathrm{mm}^{3}$ and a glucose level of $40 \mathrm{mg} / \mathrm{dl}$. At the time of admission, it was technically impossible to determine VDRL in the CSF. A later result was negative.

An MRI of the brain showed multiple lacunar ischemic lesions in the basal ganglia, enhancement of the leptomeningeal vessels in the left frontoparietal area, mild T2 and FLAIR signal hyperintensity in the left frontoparietal subcortical area. (Figure 1)

Based on clinical signs, laboratory data and the brain MRI, neurosyphilis was diagnosed.

Penicillin G was started, 24 million IU infusion/day, divided into four doses, for 14 days. After that intramuscular benzathine penicillin, 2.4 million units once per week was administered by intramuscular injection and continued for three weeks.

At follow-up, six months later, she had no symptoms, and serological tests were negative.

\section{Discussion}

Neurosyphilis is a neurologic disease underdiagnosed especially because of its atypical, and sometimes bizarre clinical features [1-3]. Osler stated in 1892 that "syphilis simulates all other diseases" and called it "the great imitator" [4]. In the elderly, neurosyphilis is often overlooked in the diagnostic algorithm of an ischemic stroke [1, 3-5]. Frequently, the onset can appear to be an ischemic stroke, dementia, or psychosis [1-3, 6-9]. Neurosyphilis is determined by Treponema pallidum infection of the brain, meninges and spinal cord, affecting approximately $25-40 \%$ of untreated infected patients [6-9]. It is caused by an infection with Treponema pallidum, appearing in the secondary or tertiary phase of the disease[3, 8-10].

In the majority of cases, it occurs 10-20 years after the initial infection, $[1,5,7]$ although it may appear after a shorter period [8-9].

The most common known clinical features of neurosyphilis are neuropsychic changes (psychosis, dementia, delirium), strokes, ophthalmologic features (uveitis, optic neuritis), myelopathies, seizures, brainstem or cranial nerves involvement [2, 3, 6, 8-10].

Neurosyphilis represents a diagnostic problem because of its nonspecific clinical features and given the recurrence of the disease both in young and elderly patients whether they are immunocompromised or not $[1,2,8,9]$.

There are many reported cases of delayed diagnosis of neurosyphilis, explained by its atypical clinical features [1-7].

The patient reported in this instance had skin lesions, most probably syphilitic gummas, which had not been diagnosed as such, and the interpretation of the cerebral lesions as shown on the CT scan of the brain as being secondary to untreated hypertension resulted in a delayed diagnosis. Establishing an accurate diagnostic was possible only after multiple

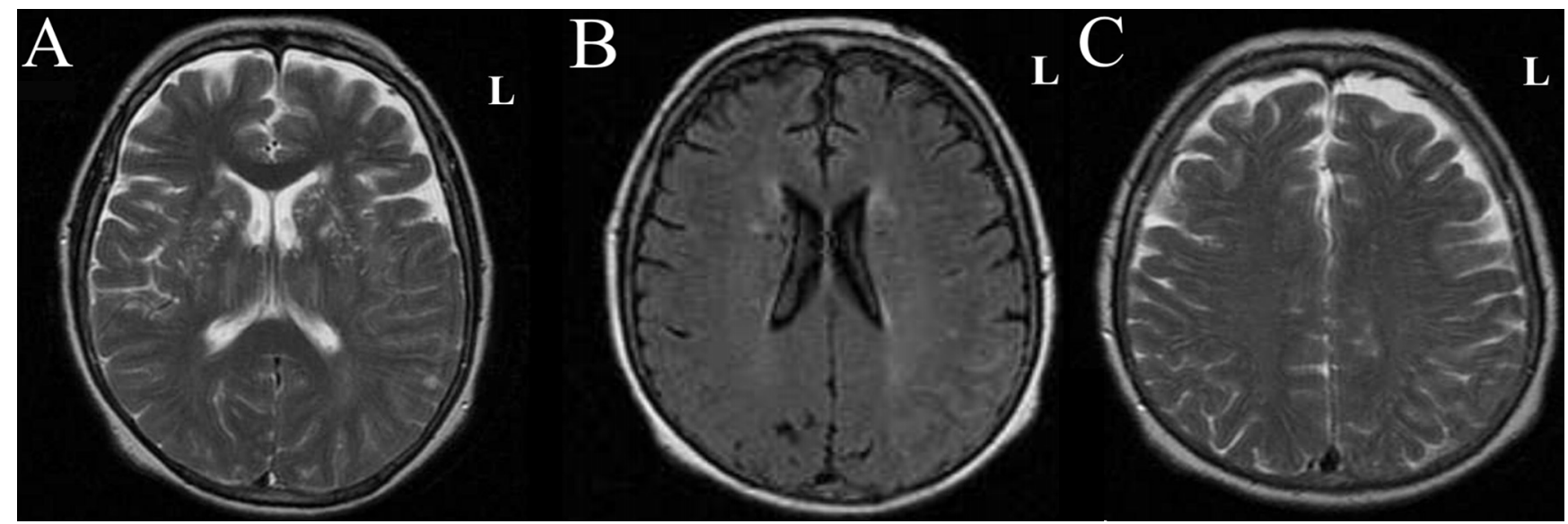

Fig. 1. A. Multiple lacunar ischemic lesions in basal ganglia, dilatation of the Virchow-Robin spaces. B. Enhancement of the leptomeningeal vessels in left frontoparietal area. C. Enhancement of the leptomeningeal vessels in left frontoparietal area, mild T2 and FLAIR signal hyperintensity in left frontoparietal subcortical area. 
investigations regarding the aetiology of an ischemic stroke.

It was impossible, for technical reasons, to determine VDRL in the CSF upon admission, and a later result was negative. However, the VDRL in the CSF is reactive in only $70-75 \%$ of cases [1], and a negative result does not exclude the diagnosis of neurosyphilis [11]. If the VDRL in the CSF is negative, a white blood cell count more than five cells $/ \mathrm{mm} 3$ or a protein level more than $46 \mathrm{mg} / \mathrm{dl}$ are suggestive of neurosyphilis [12].

The standard therapy for neurosyphilis is intravenous Penicillin G, 12-24 million units continuous IV infusion for 10-14 days followed by Benzathine penicillin, 2.4 million units intramuscular once per week for up to three weeks $[1,11]$. An alternative treatment is by intramuscular Penicillin G procaine, 2.4 million units qd, plus Probenecid $500 \mathrm{mg}$ orally four times a day for 10-14 days. After treatment, follow-up clinical and CSF examination should be scheduled at 3-6 months $[8,11]$.

Recognising and diagnosing neurosyphilis can be difficult even for an experienced physician. Because of the increasing incidence of syphilis in the last decade, consideration should be given to a diagnosis of neurosyphilis in every patient presenting with an ischemic stroke [13].

According to the literature, the common situations in clinical practice, when neurosyphilis should be considered as a possible diagnosis are young patients with ischemic stroke, elderly patients with multiple ischemic strokes despite a correct therapy, patients with progressive cognitive deterioration and patients with clinical features suggestive of neurosyphilis [3, 8-10].

\section{CONCLUSIONS}

Neurosyphilis is a disease overlooked because of its atypical, and sometimes bizarre clinical features. Classical presentation suggestive of neurosyphilis is represented by neuropsychiatric changes (psychosis, dementia, delirium), strokes, ophthalmologic features (uveitis, optic neuritis), myelopathies, seizures, brainstem or cranial nerves involvement. Elderly patients with uncharacteristic neurologic and cognitive symptoms need extensive assessment to identify the aetiology of an ischemic stroke. In every patient with an ischemic stroke, the possible presence of a neurosyphilis should be considered, and appropriate treatment should be administered. Specific antibiotic therapy can resolve the neurologic and cognitive symptoms completely.

\section{DONFLICT OF INTEREST}

None declared.

\section{DEFERENCES}

1. Madhusudhan M. Neurosyphilis. Neurol India. 2009;57:233-4.

2. Costiniuk $\mathrm{CT}$, MacPherson PA. Neurocognitive and psychiatric changes as the initial presentation of neurosyphilis. CMAJ. 2013;185:499-503.

3. Cordato DJ, Djekic S, Taneja SR, et al. Prevalence of positive syphilis serology and meningovascular neurosyphilis in patients admitted with stroke and TIA from a culturally diverse population (2005-09). J Clin Neurosci. 2013;20:943-7.

4. Tziotzios C, Walters M. Keep your eyes open: an unusual case of ptosis. Case report. QJ Med. 2011;104:257-8.

5. Umashankar G, Gupta V, Harik SI. Acute bilateral inferior cerebellar infarction in a patient with neurosyphilis. Arch Neurol. 2004;61:953-6.

6. Mehrabian S, Raycheva Radoslavova M, Petrova Petrova E, Tsankov NK, Traykov Dintchov L. Neurosyphilis presenting with dementia, chronic chorioretinitis and adverse reactions to treatment: a case report. Cases J. 2009;2:8334.

7. Simu M, Rosca EC, Chirileanu RD. Neurosyphilis- A case study. TMJ. 2007;57:157-61.

8. Tso MK, Koo K, Tso GY. Neurosyphilis in a non-HIV patient: more than a psychiatric concern. Mcgill J Med. 2008;11:160-3.

9. Tsui YK, Mok CK, Yuen MK. Recurrent stroke and neurosyphilis in an elderly patient- a case report. HK Pract. 1998;20:447-53.

10. Asdaghi N, Muayqil T, Scozzafava J, Jassal R, Saqqur M, Jeerakathil TJ. The re-emergence in Canada of meningovascular syphilis: 2 patients with headache and stroke. CMAJ. 2007;176:1699-1700.

11. Basic V, Podobnik-Sarkanji S, Seric V, Demarin V. Neurosyphilis: A Case Report. Acta Clin Croat. 2000;39:47-50.

12. Pastuszczak M, Wojas-Pelc A. Current standards for diagnosis and treatment of syphilis: selection of some practical issues, based on the European (IUSTI) and U.S. (CDC) guidelines; Postepy Dermatol Alergol. 2013;30:203-10.

13. Beauchemin P, Laforce R. Neurocognitive changes in tertiary neurosyphilis: a retrospective chart review. Can J Neurol Sci. 2014;41:452-8. 